\title{
Data-Driven Imaging in Anisotropic Media
}

\author{
Arno VOLKER ${ }^{1}$ and Alan HUNTER ${ }^{1}$ \\ TNO Stieltjesweg 1, 2600 AD, Delft, The Netherlands \\ Phone: +31 (0)88 86 66292, e-mail: arno.volker@tno.nl
}

\begin{abstract}
.
Anisotropic materials are being used increasingly in high performance industrial applications, particularly in the aeronautical and nuclear industries. Some important examples of these materials are composites, single-crystal and heavy-grained metals. Ultrasonic array imaging in these materials requires exact knowledge of the anisotropic material properties. Without this information, the images can be adversely affected, causing a reduction in defect detection and characterization performance.

The imaging operation can be formulated in two consecutive and reciprocal focusing steps, i.e., focusing the sources and then focusing the receivers. Applying just one of these focusing steps yields an interesting intermediate domain. The resulting common focus point gather (CFP-gather) can be interpreted to determine the propagation operator. After focusing the sources, the observed travel-time in the CFP-gather describes the propagation from the focus point to the receivers. If the correct propagation operator is used, the measured traveltimes should be the same as the time-reversed focusing operator due to reciprocity. This makes it possible to iteratively update the focusing operator using the data only and allows the material to be imaged without explicit knowledge of the anisotropic material parameters. Furthermore, the determined propagation operator can also be used to invert for the anisotropic medium parameters.

This paper details the proposed technique and demonstrates its use on simulated array data from a specimen of Inconel single-crystal alloy commonly used in the aeronautical and nuclear industries.
\end{abstract}

Keywords: Ultrasonic, imaging, focusing, phase array, anisotropic.

\section{Introduction}

Phased array imaging is becoming an increasingly powerful tool for characterizing the interiors of objects. Additionally phased array systems are becoming more common in use. To construct a high quality image, detailed information about the actual velocity field in a medium is required. Using an incorrect velocity yields a blurred image and may produce significant artifacts, which can lead to erroneous interpretation. This is particularly the case in anisotropic media. Anisotropic materials are being used increasingly in high performance industrial applications, particularly in the aeronautical and nuclear industries. Some important examples of these materials are composites, single-crystal and heavy-grained metals.

In this paper we introduce a generalized concept that allows the extraction of the imaging operators directly from the data, without the need to make any prior assumptions about the medium. We demonstrate the concept based on modeled finite difference data using a strongly anisotropic single crystal steel.

\section{Common focal point method}

The forward modeling procedure is conceptually explained in Figure 1, using a wave field operator formulation [1]. In Figure $1, \mathbf{S}\left(z_{0}\right)$ is the emitted wave field from a wedge transducer at the steel-wedge interface. This operator includes elastic transmission effects from the wedge into the steel. The wave field propagation operator $\mathbf{W}^{+}\left(z_{m}, z_{0}\right)$, extrapolates the wave field from the top surface to the back wall. Note that zm may vary with the lateral coordinate. 
The wave field is reflected from the back wall, where $\mathbf{R}^{+}\left(z_{m}\right)$ describes the angle dependent reflection from the backwall. The upward propagation operator $\mathbf{W}^{-}\left(z_{0}, z_{m}\right)$ propagates the waves to the outer surface at $\mathrm{z} 0$, again $\mathrm{z} 0$ may vary laterally. Part of the wave field is detected by a wedge transducer, described by $\mathbf{D}\left(z_{0}\right)$ and another part is reflected back, described by $\mathbf{R}^{-}\left(z_{0}\right)$. Each pass through the feedback loop yields another, higher order skip. The wave field description is in the temporal frequency domain.

The inversion model is parameterized with $z_{0}=z_{0}(x)$ and $z_{m}=z_{m}(x)$, where $\mathrm{x}$ is the lateral coordinate. The data matrix for primary reflections, i.e., ignoring multiple reflections can be written as:

$$
\mathbf{P}\left(z_{0}\right)=\mathbf{D}\left(z_{0}\right) \mathbf{W}^{-}\left(z_{0}, z_{m}\right) \mathbf{R}^{+}\left(z_{m}\right) \mathbf{W}^{+}\left(z_{m}, z_{0}\right) \mathbf{S}\left(z_{0}\right)
$$

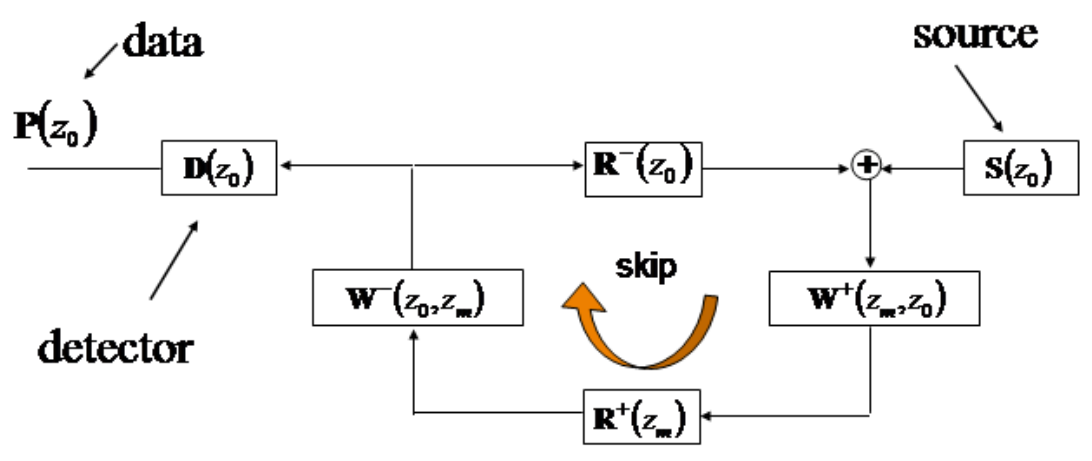

Figure 1 Schematic illustration of that described wave propagation in elastic, anisotropic media, The wave field is being generated by an arbitrary source array $(\mathrm{S})$ that both describes the directivity and the signature. The wave field propagates down $\left(\mathrm{W}^{+}\right)$, gets reflected $\left(\mathrm{R}^{+}\right)$, where the reflection operator describes general angle dependent reflectivity. The second propagation $\left(\mathrm{W}^{-}\right)$is back to the surface and the detector response is expressed by D. Multiple reflections are generated by the feedback loop.

Imaging can be formulated in terms of two consecutive focusing steps [2,3], focusing the sources and then focusing the receivers. The focusing operator for the sources is defined as:

$$
\mathbf{W}^{+}\left(z_{m}, z_{0}\right) \mathbf{S}\left(z_{0}\right) F\left(z_{m}, z_{0}\right)=\delta_{j}\left(z_{m}\right),
$$

and for the receivers:

$$
F_{i}^{T}\left(z_{m}, z_{0}\right) \mathbf{D}\left(z_{0}\right) \mathbf{W}^{-}\left(z_{0}, z_{m}\right)=\delta_{i}^{T}\left(z_{m}\right)
$$

The bold symbols indicate matrices, while the normal font with a subscript indicates a vector. The vector $\delta_{j}\left(z_{m}\right)$ indicates a band limited delta function, with essentially one non-zero element at location $\mathrm{j}$. These focusing operators are actually vectors, where the symbol ' $\mathrm{T}$ ' indicates that the vector is a row vector. Applying this to the data matrix yields:

$$
\begin{aligned}
F_{i}^{T}\left(z_{m}, z_{0}\right) \mathbf{P}\left(z_{0}\right) F_{j}\left(z_{0}, z_{m}\right) & =F_{i}^{T}\left(z_{m}, z_{0}\right) \mathbf{D}\left(z_{0}\right) \mathbf{W}^{-}\left(z_{0}, z_{m}\right) \mathbf{R}\left(z_{m}\right) \mathbf{W}^{+}\left(z_{m}, z_{0}\right) \mathbf{S}\left(z_{0}\right) F_{j}\left(z_{0}, z_{m}\right) . \\
& =R_{i j}\left(z_{m}\right)
\end{aligned}
$$


This procedure extracts one element from the reflection matrix. Normally confocal imaging is used, i.e., $i=j$.

If just one focusing step is applied, an interesting intermediate domain is obtained. The result is called the common focus point gather (CFP):

$$
P_{j}\left(z_{0}, z_{m}\right)=\mathbf{P}\left(z_{0}\right) F_{j}\left(z_{0}, z_{m}\right)=\mathbf{D}\left(z_{0}\right) \mathbf{W}^{-}\left(z_{0}, z_{m}\right) \mathbf{R}\left(z_{m}\right) \delta_{j}\left(z_{m}\right) .
$$

The travel time of the common focus point response is from the target point to the receiver positions at the surface. While the focusing operator contains the travel time for the source locations at the surface to the target point. In the focusing operator, these travel times are the delays that have to be applied to ensure that the waves generated by the source arrive at the same time at the target location. So comparing the observed travel time with the time reversed focusing operator allows us to perform a consistency check. The differential time shift (DTS) panel shows the travel time difference between the CFP-gather and the focusing operator. The observed difference can be used to update the focusing operator. We will demonstrate this concept using numerically modeled data.

\section{Modeled data using anisotropic finite difference method}

The technology outlined in the previous paragraphs is demonstrated on numerically modeled data. The model consists of a 64 element phased array, where all elements are fired sequentially (see Figure 2).

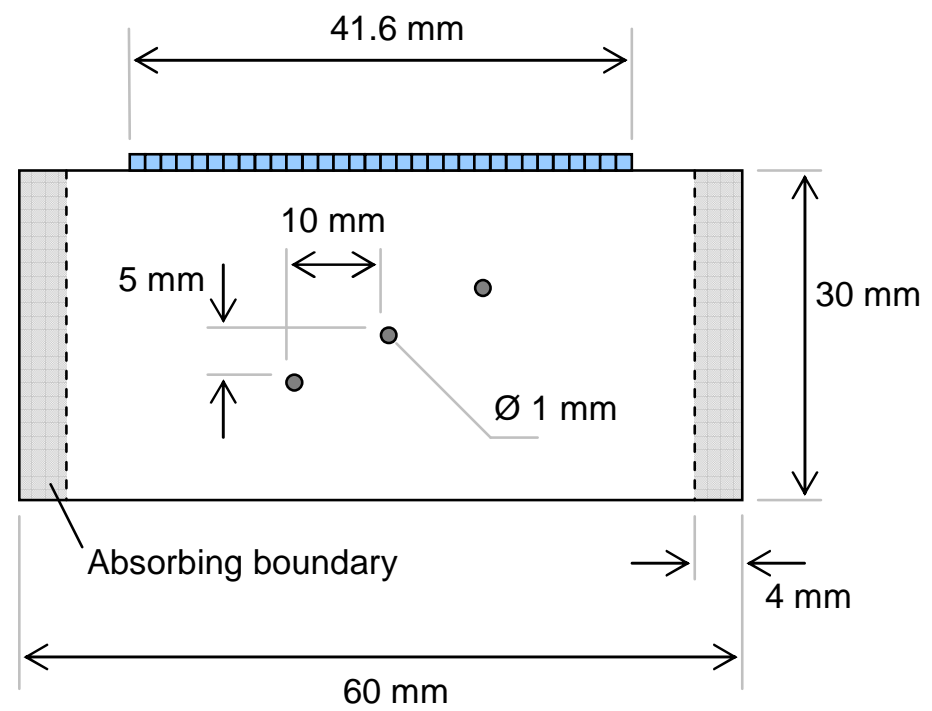

Figure 2 The model geometry, consisting of a 64 element phased array and a strongly anisotropic metal with three side-drilled holes. The propagation velocity in the $0^{\circ}$ and $90^{\circ}$ direction is $90 \%$ of that in the $45^{\circ}$ direction.

The resulting wave field is recorded by all elements. The medium is homogeneous, but strongly anisotropic and contains three side drilled holes. The left and right side of the model are absorbing regions to avoid reflections from these boundaries. As reference, the same geometry is used to generate an isotropic dataset.

Two snapshots of the propagating wave field are shown in Figure 3, on the left hand side for the isotropic case and on the right hand side for the anisotropic case. Comparison clearly shows the effect of the material anisotropy. The wave front travels much faster (about 10\% velocity 
difference) in the $45^{\circ}$ direction than in the $0^{\circ}$ and $90^{\circ}$ direction. Therefore the response from the side drilled holes gives strong non-hyperbolic events.

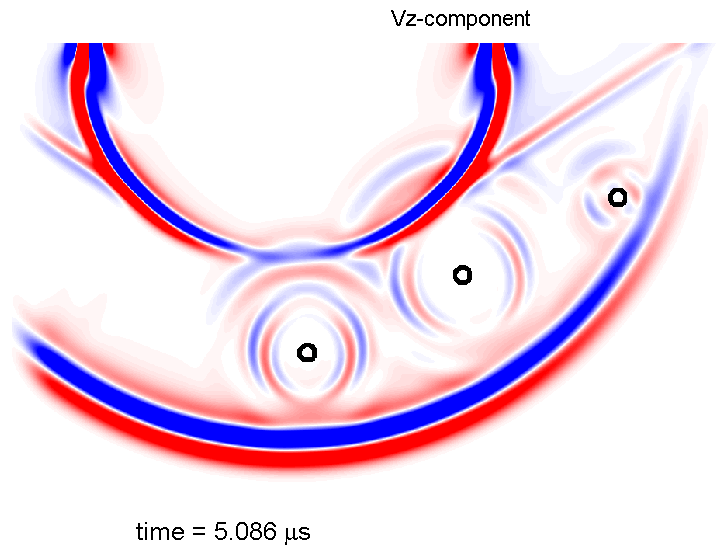

a)

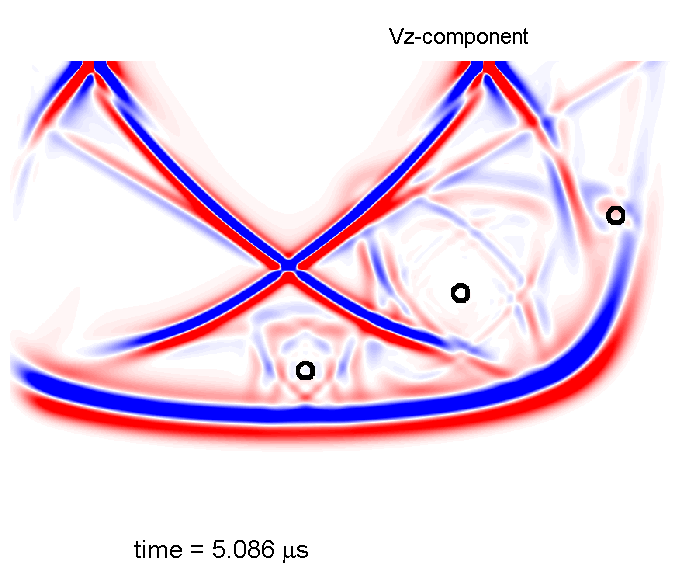

b)

Figure 3 Snap shots of the vertical component of the wave field, a) isotropic case, b) anisotropic case

A full dataset was modeled by firing each element separately and recording the wave field using all elements. Selecting only the pulse-echo traces from the full dataset, clearly shows the difference between the data from the isotropic and anisotropic models. This is shown in FIGURE 4.
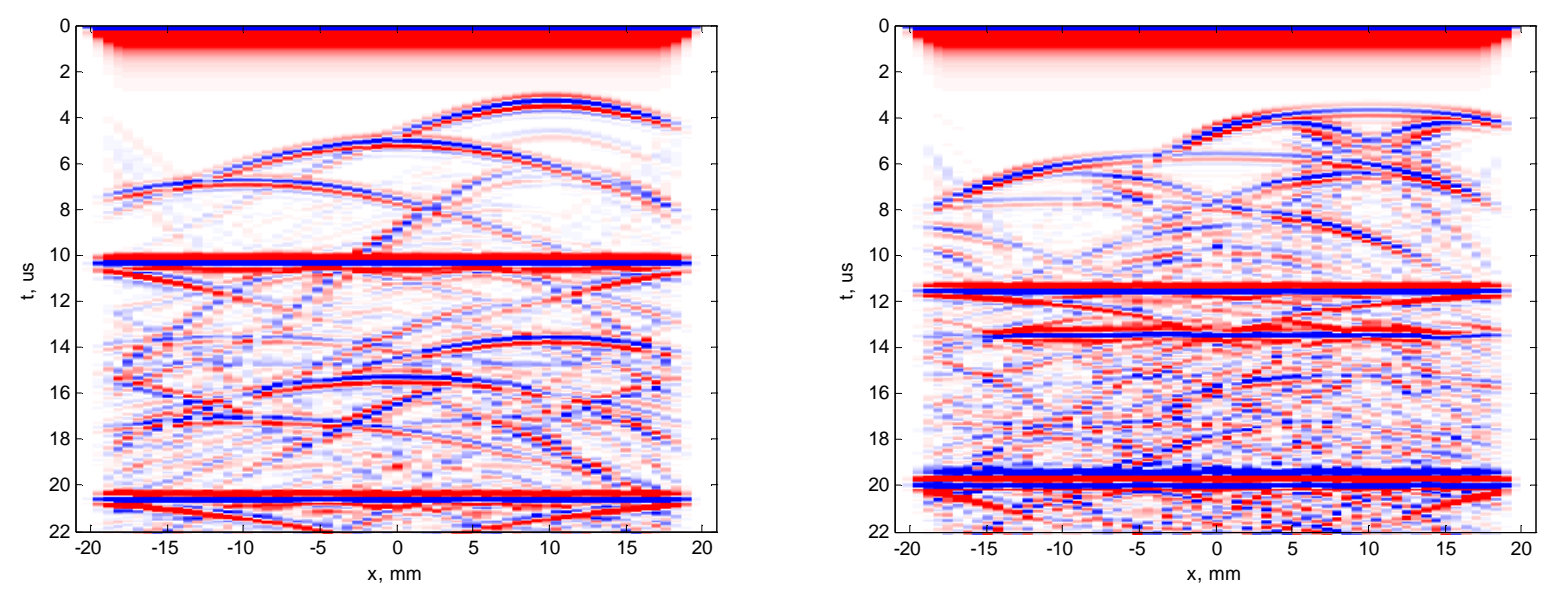

FIGURE 4 Pulse-echo section from the full data set (diagonal elements from the data matrix), a) isotropic case, b) anisotropic case.

\section{Data-driven imaging}

If a conventional imaging algorithm is applied to both datasets, making the assumption that the medium is isotropic, the images shown in Figure 5 are obtained. The isotropic image (Figure 5a) clearly indicates the three side drilled holes. For reference the position of each side drilled hole is indicated by ' $o$ '. Due to aperture limitations the indication from the first and third side drill hole are tilted. The side drilled hole on the left hand side, is mainly illuminated from the right. This explains the tilt of the indication. The same holds for the side drilled hole on the right hand side, which is mainly illuminated from the left. 
The image using the anisotropic data is quite poor, containing many artifacts. Additionally the side drilled holes are not imaged at their correction locations. Consequently, defects may be missed in the presence of structural echoes.

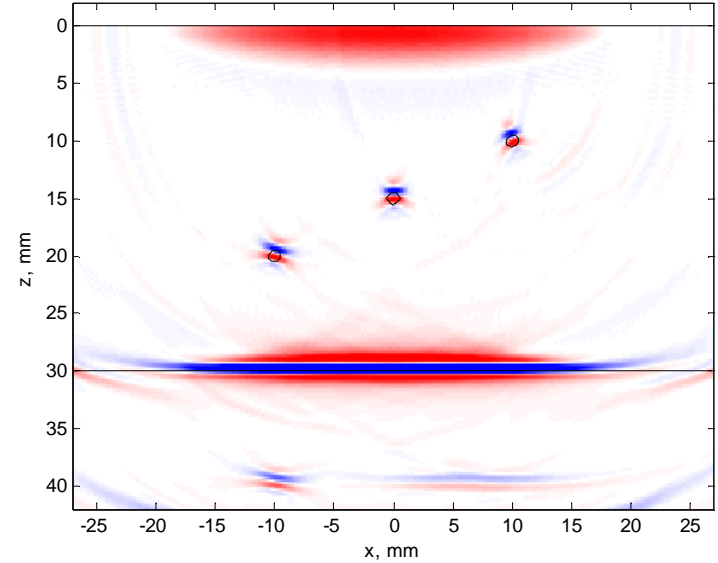

a)

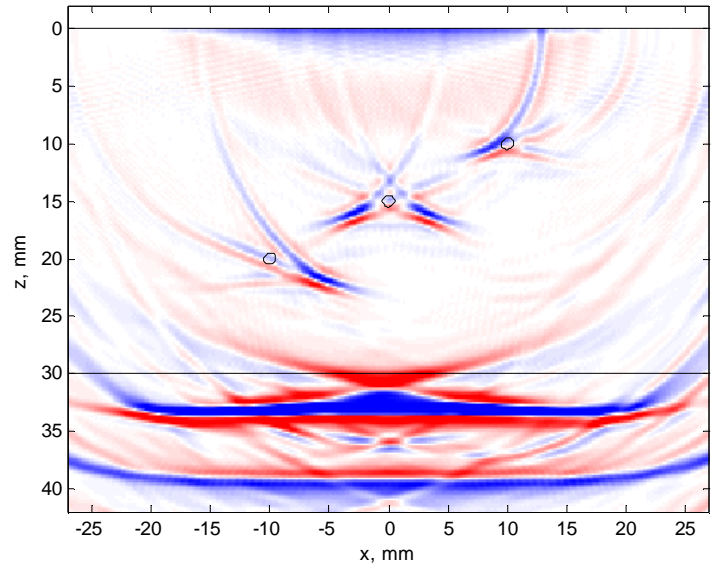

b)

Figure 5 Images assuming a isotropic medium, a) isotropic dataset, b) anisotropic dataset. The isotropic image is nearly perfect, while the isotropic image contains a lot of artifacts.

We demonstrate the approach outlined in this paper on the anisotropic dataset. First a target point on the back wall is selected (see Figure 6a). As a first guess, a hyperbolic operator is used, where the arrival time of the apex equals half the travel time of the pulse-echo signal. The DTS-panel is shown in Figure 6b for the first iteration. Using an initial operator as explained, part of the DTS panel will be at zero time, making it easy to identify the correct event to pick. Clearly the operator is not correct as can be seen from the non-zero time difference. Half the time difference is used to update the focusing operator and after a few iterations the result in Figure $6 \mathrm{c}$ is obtained. In that case the event appears at zero traveltime, which indicates that the correct focusing operator has been found

The same procedure is repeated for other points on the back wall and the response from the side drilled holes. Figure 7 demonstrates the same approach being applied to the middle side drilled hole. Again after just a few iterations a flat DTS panels is obtained as illustrated in Figure 7c. The method is very fast and can easily be applied in a user interactive fashion.

In the current example, the medium is laterally invariant; this makes it fairly straightforward to interpolate the focusing operators to a full set, which can be used to generate an image. The result of this procedure is shown in Figure 8b. For reference the original image is shown in Figure $8 \mathrm{a}$. The result shows that we have successfully imaged the three side drilled holes. The resolution in the image is slightly lower compared to the isotropic case because of the anisotropy; this is due to the fast velocity in the $45^{\circ}$ direction causing the diffraction signals to have less curvature and hence yield a lower resolution. 


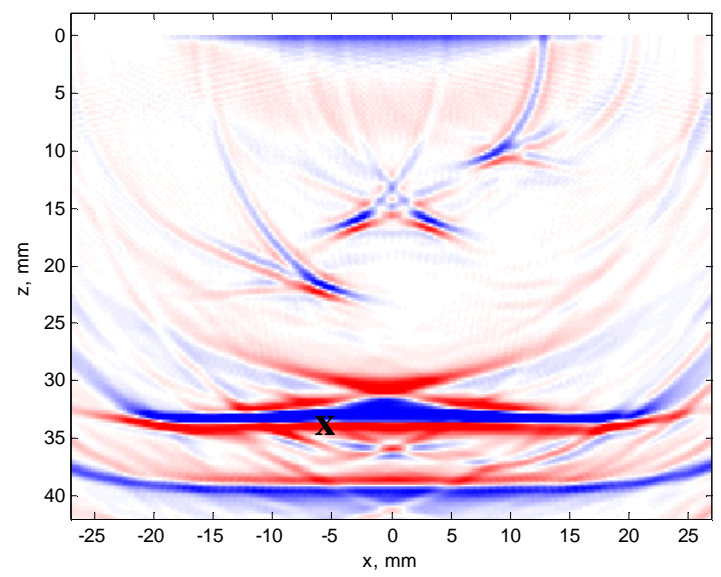

a)

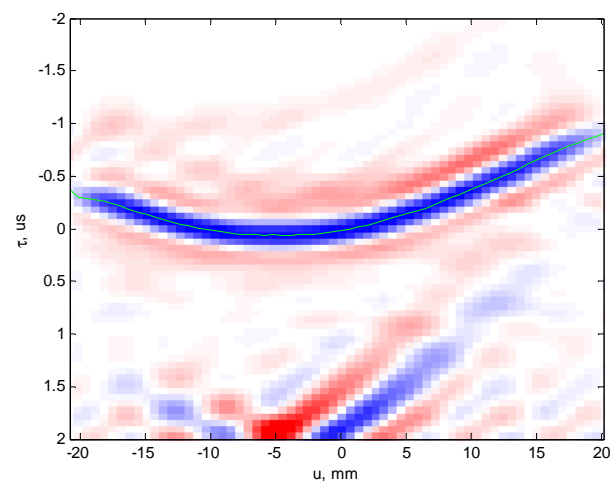

b)

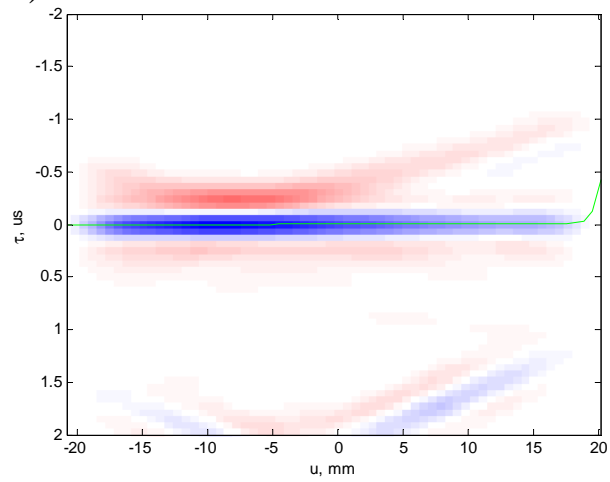

c)

Figure 6 Illustration of the focusing operator updating procedure for a point on the backwall, a) shows the initial image with the target point indicated by ' $x$ ', $b$ ) shows the initial DTS-panel and c) shows the final DTS-panel after just a few iterations.

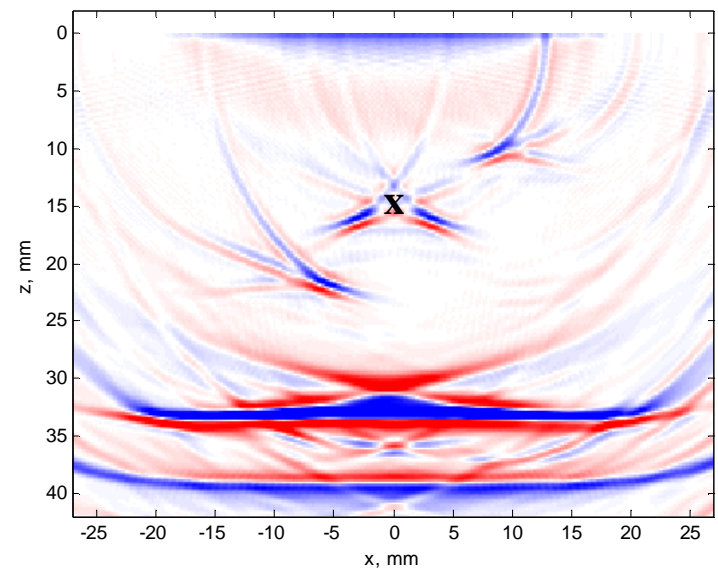

a)

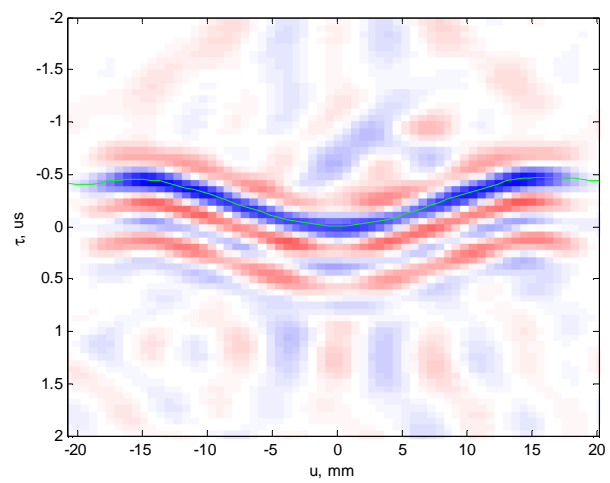

b)

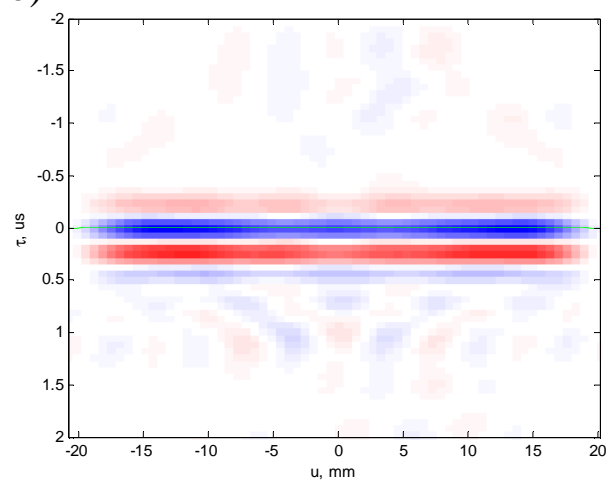

c)

Figure 7 Illustration of the focusing operator updating procedure on the middle side drilled hole, a) shows the initial image, b) shows the initial DTS-panel and c) shows the final DTSpanel after just a few iterations. 


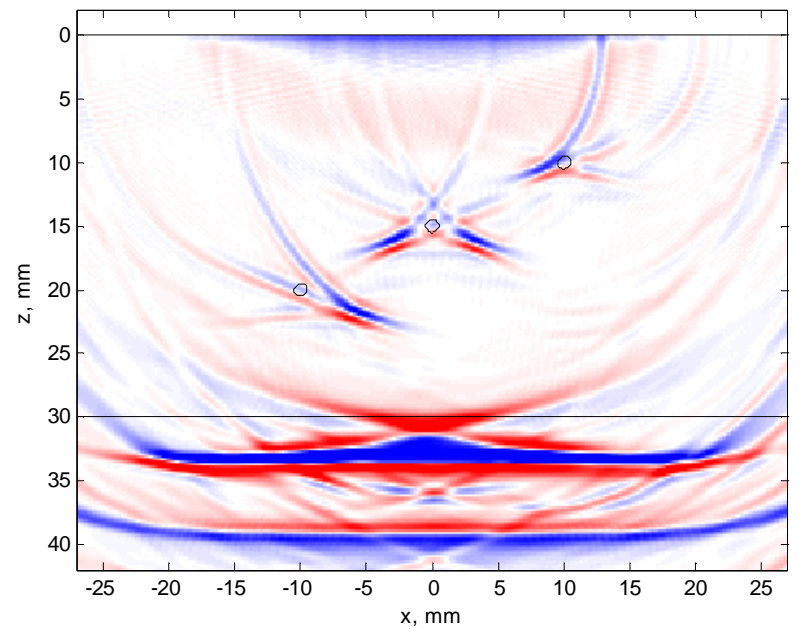

a)

Figure 8 Comparison of original image and the one obtained by applying the interpolated focusing operators from the data.

\section{Operator inversion}

In more complicated media, for example an austenitic weld, the operator interpolation procedure is not straightforward. In that case a tomographic inversion algorithm can be used to invert for the medium properties using the derived operators. In general the location of the focus point and the medium properties are unknown. Inverting the focusing operators allows one to estimate both. Figure 9a shows the inverted angular velocity in the medium. Based on this, a focusing operator table can be calculated as shown in Figure 9b. The resulting image is shown in Figure 9c. Comparison with Figure 8b shows essentially no difference in image quality. 


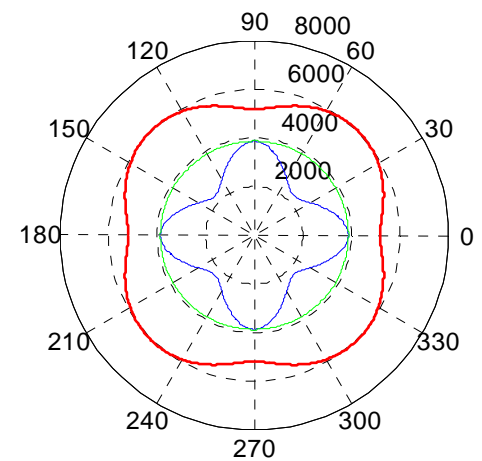

a)

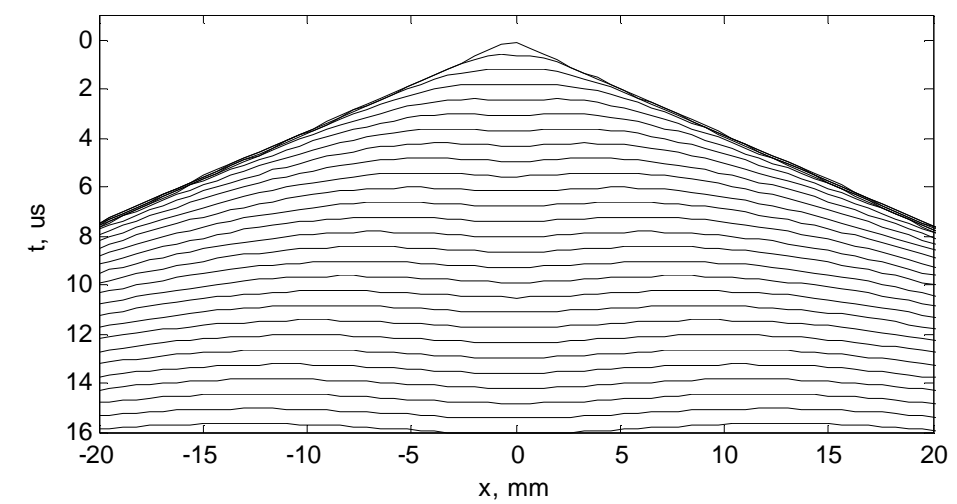

b)

Figure 9 Illustration of operator inversion process, a) first the velocity model is obtained by using an appropriate inversion scheme, b) based on the velocity model a set of focusing operators is calculated. These are then used to construct an image (c).

\section{Conclusions}

This paper has demonstrated a new technique to determine focusing operators from ultrasonic data. The concept was successfully applied to a modeled dataset for a strongly anisotropic medium. Extracting the focusing operators is a straightforward process, which can be performed interactively. For laterally invariant media, the operators can be interpolated very simply. For more complicated media, the operators need to be inverted. This yields a spatially variant velocity field and the velocity model can then be used to calculate the focusing operators.

The proposed method does not make any assumptions regarding the medium or the wave type. This means it can also be applied to shear waves or guided waves. Moreover extension to 3D data from 2D phased arrays is straightforward. 


\section{References}

1. A.J. Berkhout, Seismic migration; A. Theoretical aspects, Elsevier sciences publishers, Amsterdam, 1985.

2. A.J. Berkhout, 1997a, Pushing the limits of seismic imaging, part I: Prestack migration in terms of double dynamic focusing: Geophysics, 62,937-953.

3. A.J. Berkhout, 1997b, Pushing the limits of seismic imaging, part II: Integration of prestack migration, velocity, estimation, and AVO analysis: Geophysics, 62, 954969. 